\title{
Cardinality Constrained Robust Optimization Applied to a Class of Interval Observers
}

\author{
Philip James McCarthy Christopher Nielsen Stephen L. Smith
}

\begin{abstract}
We propose a linear programming-based method of interval observer design for systems with uncertain but bounded model parameters and initial conditions. We assume that each uncertain parameter in the system model is bounded by conservative guaranteed bounds, and tighter conditional bounds. We define a class of systems by the number of conservative bounds required to bound all uncertain parameters. Using robust optimization, we solve a single linear program per class of systems to obtain gains for the interval observer. A conservative upper bound on the worst-case steady-state performance of the interval observers over the specified class of systems is minimized.
\end{abstract}

\section{INTRODUCTION}

In scenarios such as high-volume manufacturing of lowcost devices, there are many systems characterized by similar dynamics, whose parameters vary within known bounds. Each system is characterized by the same dynamical model with uncertain parameters. We consider scenarios wherein the bounds on specific parameters can be tightened for certain systems. For example, if higher-quality components are used to construct a batch of devices. We address the problem of state estimator design for such systems. We propose a method of optimal interval observer $(I O)$ design using robust optimization. We define a class of systems using the number of conservative bounds required to bound all uncertain parameters of the system dynamics. Only a single optimization is performed per class of systems.

An IO comprises an upper and lower observer, whose trajectories bound those of the system states from above and below, respectively. IOs are useful when the system dynamics are highly uncertain, making classic observers (e.g., Luenberger, high-gain) unreliable. One of the earliest dynamical IOs was described in [1], where they were designed for a wastewater treatment management system. IOs have also been applied to population dynamics [1], algae cultures [2], and pharmacokinetics [3]. IOs are attractive in biotechnological applications due to the large parametric and measurement uncertainty inherent to biological systems. IOs can be applied to a large class of dynamical systems, e.g., any system with bounded state trajectories [1], and any stable linear system with additive disturbances, by using a timevarying change of coordinates [4]. Necessary and sufficient conditions for the existence of IOs, yielding systematic optimal design procedures, were identified for positive linear

This research is partially supported by the Natural Sciences and Engineering Research Council of Canada (NSERC).

The authors are with the Dept. of Electrical and Computer Engineering, University of Waterloo, Waterloo ON, N2L 3G1 Canada. \{philip. mccarthy; cnielsen; stephen.smith\} @uwaterloo.ca systems in terms of matrix inequalities in [5], and for a more general class of nonlinear systems with bounded uncertainties in terms of linear constraints in [6]. The linear constraints identified in [6] were used to develop the linear programs (LPs), which we extend in this paper. Specifically, we account for uncertainty in the coefficients of these LPs.

Given an instance of a convex optimization problem with uncertain coefficients, if even relatively small deviations from nominal coefficient values, called perturbations, are not accounted for, classic optimization methods may generate solutions that are far-from-optimal, or even infeasible when implemented [7]. Robust optimization generates deterministically feasible solutions under deterministic set-based models of uncertainty at the expense of increased cost in the nominal problem. Associated with each model of uncertainty is a budget of uncertainty [7], which characterizes the class of problem instances for which the robust solution will be deterministically feasible, as well as the potential deviation from optimality of the solution to the nominal problem. For LPs, several models of uncertainty have been considered. The ellipsoidal [8] and norm [9] models of uncertainty consider various norms of perturbed vectors and matrices of coefficients. Notwithstanding a special case in [9], the robust problem formulations under these models of uncertainty are second order cone programs. The cardinality constrained model of uncertainty [10], used in this paper, considers the cardinality of coefficients that are perturbed from their nominal values. The robust formulation is implemented as an LP. Robust optimization has seen varied application, including antenna [11] and circuit [12] design, constrained stochastic linear-quadratic control [13], and wireless channel power control [14]. The reader is referred to [7] for a comprehensive review of the robust optimization literature.

We propose a method of IO design using the linear programming-based method of [6] and the robust optimization method of [10]. We define a class of systems in terms of the number of perturbed coefficients in the robust formulation of the LP of [6]. The designer can tune the robustness of the solution to improve the bounds on the steady-state error of the proposed IO.

The contributions of this paper are: 1) a novel application of cardinality constrained robust optimization to the design of dynamical observers; 2) a tunably robust interval observer design method; 3) an empirical study of the proposed observer's performance. 


\section{A. Notation and Terminology}

Given a matrix $M \in \mathbb{R}^{m \times n}$, the notation $M_{j}$ denotes the $j$ th column of $M$ and $M^{-\top}$ is the transpose of its inverse. Given two vectors $v_{1} \in \mathbb{R}^{m}$ and $v_{2} \in \mathbb{R}^{n}$, define the column vector $\operatorname{col}\left(v_{1}, v_{2}\right):=\left[\begin{array}{ll}v_{1}^{\top} & v_{2}^{\top}\end{array}\right]^{\top} \in \mathbb{R}^{m+n}$; the col function extends to an arbitrary number of arguments; applied to a matrix $M \in \mathbb{R}^{m \times n}, \operatorname{col}(M):=\operatorname{col}\left(M_{1}, \ldots, M_{n}\right) \in \mathbb{R}^{m n}$. The Kronecker delta $\delta_{i j}$ equals 0 if $i \neq j$, and 1 if $i=j$. A matrix $M \in \mathbb{R}^{n \times n}$ is said to be Metzler if all its off-diagonal elements are nonnegative. Given a matrix $M \in \mathbb{R}^{n \times n}$ and a locally Lipschitz function $\xi: \mathbb{R}^{n} \times \mathbb{R} \rightarrow \mathbb{R}^{n}$, the dynamical system $\dot{x}(t)=M x(t)+\xi(x, t)$ is said to be positive if $x(0) \geq$ 0 implies $x(t) \geq 0$ for all $t \geq 0$. When applied to vectors or matrices, the relations $>,<, \geq, \leq$ are taken elementwise. Given matrices $A \in \mathbb{R}^{m \times n}$ and $B \in \mathbb{R}^{m \times n}$, the relation $A \preceq B$ is defined as $A \leq B$, with $A_{i j}<B_{i j}$ for at least one pair $(i, j)$. Given a scalar $c \in \mathbb{R}$, vector $v \in \mathbb{R}^{n}$, or matrix $M \in \mathbb{R}^{n \times n}$, the operator $|\cdot|$ is the elementwise absolute value. Given a set $S$, its cardinality is denoted by $|S|$. We define $\mathbf{n}:=\{1, \ldots, n\}$.

\section{Problem Statement and Proposed ApProACH}

\section{A. Problem Statement}

We consider systems of the form

$$
\dot{x}=A x+\xi(x, t), \quad y=C x, \quad x(0)=x_{0},
$$

where $x \in \mathbb{R}^{n}$ is the state vector, $A \in \mathbb{R}^{n \times n}, \xi: \mathbb{R}^{n} \times$ $\mathbb{R} \rightarrow \mathbb{R}^{n}, y \in \mathbb{R}^{p}$ is the output, and $C \in \mathbb{R}^{p \times n}$. We assume existence and uniqueness of solutions for $x(t)$ for all $t \geq 0$. We impose the following standing assumptions on (1).

Assumption 1 (Boundedness of Solutions). There exists a known constant $\varkappa \in \mathbb{R}^{n}$ such that $\varkappa \geq\left|\sup _{t \geq 0} x(t)\right|$.

Assumption 2 (Uncertainty in Initial Conditions). There exist known constants $\bar{x}_{0}, \underline{x}_{0} \in \mathbb{R}^{n}$, such that $\underline{x}_{0} \leq x_{0} \leq \bar{x}_{0}$.

Assumption 3 (Uncertainty in Nonlinear Dynamics). There exist known constants $\bar{\xi}, \xi \in \mathbb{R}^{n}$, such that $\xi \leq \xi(x, t) \leq \bar{\xi}$, for all $t \geq 0$ and all $-\varkappa \leq x \leq \varkappa$.

Assumption 4 (Uncertainty in System Matrix). There exist known matrices $\underline{A}, \underline{A}^{\uparrow}, \bar{A}^{\downarrow}, \bar{A} \in \mathbb{R}^{n \times n}$, such that $\underline{A} \leq$ $\underline{A}^{\uparrow} \leq \bar{A}^{\downarrow} \leq \bar{A}$ and $\underline{A} \leq A \leq \bar{A}$. Further, $A_{i j} \leq \bar{A}_{i j}^{\downarrow}$ and $A_{u v} \geq \underline{A}_{u v}^{\uparrow}$ holds for a subset of pairs $(i, j),(u, v)$. The pairs $(i, j),(u, v)$ for which these inequalities hold are unknown at design time, but the number of pairs for which they do not hold is known.

The matrices $(\bar{A}, \underline{A})$, and $\left(\bar{A}^{\downarrow}, \underline{A}^{\uparrow}\right)$ are called the outer bounds and inner bounds of $A$, respectively.

Definition II.1. System (1) belongs to uncertainty class $\mathcal{U}(\mathfrak{u}, \mathfrak{l})$ if there exist matrices $\left(A^{u}, A^{l}\right)$ such that $A^{l} \leq A \leq$ $A^{u}, \bar{A}^{\downarrow} \leq A^{u} \leq \bar{A}$, and $\underline{A} \leq A^{l} \leq \underline{A}^{\uparrow}$ with at most $\mathfrak{u}$ elements of $A^{u}$ satisfying $A_{i j}^{u}>\bar{A}_{i j}^{\downarrow}$ and at most $\mathfrak{l}$ elements of $A^{l}$ satisfying $A_{u v}^{l}<\underline{A}_{u v}^{\uparrow}$.

The class $\mathcal{U}(\mathfrak{u}, \mathfrak{l})$ contains all systems for which $A$ can be upper bounded using at most $\mathfrak{u}$ outer bounds from $\bar{A}$, and lower bounded using at most $\mathfrak{l}$ outer bounds from $\underline{A}$.
An interval observer $(I O)$ comprises an upper observer with state estimates $\hat{x}^{u}$ that bounds the true states $x$ from above, and a lower observer with state estimates $\hat{x}^{l}$ that bounds the true states from below, i.e., for all $t \geq 0, \hat{x}^{l}(t) \leq x(t) \leq$ $\hat{x}^{u}(t)$. This is called the interval property, and for it to be satisfied, the interval error $e:=\hat{x}^{u}-\hat{x}^{l}$ must satisfy positivity. If the evolution of the interval error is governed by positive dynamics, then by Assumption 2 we can choose $\left(\hat{x}^{u}(0), \hat{x}^{l}(0)\right):=\left(\bar{x}_{0}, \underline{x}_{0}\right)$ to satisfy the interval property.

Problem II.2. Given constants $\mathfrak{u}$ and $\mathfrak{l}$, design an IO whose maximum steady-state interval error, over the uncertainty class $\mathcal{U}(\mathfrak{u}, \mathfrak{l})$, is minimized with respect to the $\ell_{1}$-norm, i.e., $\|\bar{e}\|_{1}:=\limsup _{t \rightarrow \infty}\|e(t)\|_{1}$.

In this problem we know only the class $\mathcal{U}(\mathfrak{u}, \mathfrak{l})$ when designing the IO. At runtime, to implement the observer, we must know a pair of matrices $\left(A^{u}, A^{l}\right)$ satisfying Definition II.1 to properly define the observer's dynamics to satisfy the interval property.

\section{B. Proposed Approach}

Solving Problem II.2 is challenging; even in the simplest case where all outer bounds must be used, i.e., $\mathfrak{u}, \mathfrak{l}=n^{2}$, only approximate solutions are known [6, Theorem 4.2]. We too solve an approximation of Problem II.2, drawing upon the theory of [6] and [10]. As explained in Section IV$\mathrm{B}$, we consider an uncertainty class generated by how the uncertainty in $A$ appears in the proposed IO design LP; this class contains conservative characterizations of uncertainty that are not physically realizable. Consequently, we minimize a conservative upper bound on $\|\bar{e}\|_{1}$, which is at least as great as the maximum $\|\bar{e}\|_{1}$ over the uncertainty class.

We use the linear programming-based IO design method of [6] to address optimality, and apply the robust optimization method of [10] to optimize over the given uncertainty class.

\section{BACKGROUND}

\section{A. Linear Programming-Based Interval Observers}

In [6], an IO is constructed for system (1) with dynamics

$$
\begin{aligned}
& \dot{\hat{x}}^{u}=\bar{A} \hat{x}^{u}+L\left(y-C \hat{x}^{u}\right)-(\bar{A}-\underline{A}) \phi\left(\hat{x}^{u}\right)+\bar{\xi} \\
& \dot{\hat{x}}^{l}=\bar{A} \hat{x}^{l}+L\left(y-C \hat{x}^{l}\right)-(\bar{A}-\underline{A}) \psi\left(\hat{x}^{l}\right)+\underline{\xi} \\
& \left(\hat{x}^{u}(0), \hat{x}^{l}(0)\right)=\left(\bar{x}_{0}, \underline{x}_{0}\right),
\end{aligned}
$$

where $\psi(x):=\frac{1}{2}(x+|x|)\left[\phi(x):=\frac{1}{2}(x-|x|)\right]$ retains the positive [negative] elements of $x$ and maps the negative [positive] elements to 0 .

System (2) is an IO for (1), if and only if the following LP is feasible [6, Theorems 3.1, 4.3].

\section{Problem III.1.}

$$
\begin{aligned}
\text { minimize: } & {[2(\bar{A}-\underline{A}) \varkappa+\bar{\xi}-\underline{\xi}]^{\top} \lambda } \\
\text { subject to: } & \bar{A}^{\top} \lambda-C^{\top} Z \mathbf{1}_{n}=-\mathbf{1}_{n} \\
& \underline{A}^{\top} \operatorname{diag}(\lambda)-C^{\top} Z+\beta I \geq \mathbf{0}_{n \times n} \\
& \lambda>\mathbf{0}_{n}
\end{aligned}
$$

The gain matrix $L$ of (2) is defined as

$$
L:=\operatorname{diag}(\lambda)^{-1} Z^{\top} .
$$


The first and second constraints ensure that $(A-L C)$ is Hurwitz for all $A \leq \bar{A}$, and Metzler for all $A \geq \underline{A}$, thereby ensuring stability and positivity of the linear interval error dynamics, respectively. We refer to the first and second constraints as the Hurwitz and Metzler constraints, respectively. The third constraint relates to the first two, and derives from positive linear systems theory [6]. In implementation, we constrain $\lambda \geq \varepsilon \mathbf{1}_{n}$, where $\varepsilon>0$ is arbitrarily small, so the feasible region is closed.

The cost function of Problem III.1 is an upper bound on the $\ell_{1}$-norm of the steady-state supremum of the interval error [6, Theorem 4.2],

$$
\|\bar{e}\|_{1} \leq-[2(\bar{A}-\underline{A}) \varkappa+\bar{\xi}-\underline{\xi}]^{\top}(\bar{A}-L C)^{-\top} \mathbf{1}_{n} .
$$

\section{B. Cardinality Constrained Robust Optimization}

In [10], a method is proposed for protecting against varying numbers of perturbed coefficients, given an LP of the following form.

\section{Problem III.2.}

$$
\begin{aligned}
\text { minimize: } & c^{\top} q \\
\text { subject to: } & E q \leq b \\
& l \leq q \leq u,
\end{aligned}
$$

where $E \in \mathbb{R}^{m \times n}, b \in \mathbb{R}^{m}$, and $q, l, u, c \in \mathbb{R}^{n}$.

We assume that only $E$ is uncertain. The set $J_{i}, i \in \mathbf{m}$, contains the indices of the uncertain coefficients in constraint $i$. If $j \in J_{i}, i \in \mathbf{m}$, then the $j$ th coefficient of the $i$ th constraint lies in the interval $\left[E_{i j}-\widehat{E}_{i j}, E_{i j}+\widehat{E}_{i j}\right]$. The values of the perturbation terms $\widehat{E}_{i j} \geq 0$ are known for all $i, j$. The protection levels and $\Gamma_{i} \in \mathbb{Z}_{>0}{ }^{1}, i \in \mathbf{m}$, specify the number of perturbed coefficients to protect against in the $i$ th constraint. The vector $\Gamma:=\operatorname{col}\left(\Gamma_{1}, \ldots, \Gamma_{m}\right)$ specifies only the cardinalities of the sets of protected coefficients - it does not specify individual coefficients to be protected.

The robust formulation of Problem III.2, as developed in [10], is given in Problem III.3.

\section{Problem III.3.}

$$
\begin{aligned}
\text { minimize: } & c^{\top} q \\
\text { subject to: } & \sum_{j=1}^{n} E_{i j} q_{j}+\Omega_{i}\left(q, \Gamma_{i}\right) \leq b_{i} \quad i \in \mathbf{m} \\
& l \leq q \leq u,
\end{aligned}
$$

where, for $i \in \mathbf{m}$,

$$
\Omega_{i}\left(q, \Gamma_{i}\right):=\max _{\left\{S_{i}\left|S_{i} \subseteq J_{i},\right| S_{i} \mid \leq \Gamma_{i}\right\}}\left\{\sum_{j \in S_{i}} \widehat{E}_{i j}\left|q_{j}\right|\right\} .
$$

If $q_{j}>0\left[q_{j}<0\right]$, then $E_{i j}$ is perturbed in the positive [negative] direction. Notice that $\Omega_{i} \geq 0, i \in \mathbf{m}$. The set $S_{i}$ contains the indices of perturbed coefficients in constraint $i$. Maximizing over $S_{i}$ identifies the set of coefficients whose perturbation maximizes the left-hand side of constraint $i$.

If $\Gamma_{i}=0, i \in \mathbf{m}$, then $\Omega_{i}\left(q, \Gamma_{i}\right)=0, i \in \mathbf{m}$, and Problem III.3 reduces to Problem III.2.

${ }^{1}$ We restrict $\Gamma_{i}$ to $\mathbb{Z}_{\geq 0}$, but extension to $\mathbb{R}_{\geq 0}$ is straightforward [15].
Problem III.3 has an equivalent linear formulation [10, Theorem 1], which is used for computations.

\section{ROBUST INTERVAL OBSERVERS}

If the inner bounds $\left(\bar{A}^{\downarrow}, \underline{A}^{\uparrow}\right)$ are used in Problem III.1 instead of the outer bounds $(\bar{A}, \underline{A})$, then the set of feasible solutions is enlarged, thereby potentially reducing the attainable minimum cost. However, the inner bounds cannot be used unless $\underline{A}^{\uparrow} \leq A \leq \bar{A}^{\downarrow}$. By casting Problem III.1 in the framework of Problem III.3, we develop a tunably robust interval observer $(R I O)$. The designer specifies the robustness of the solution via a tuning parameter, defined by the number of elements of $A$ that violate $A_{i j} \leq \bar{A}_{i j}^{\downarrow}$, and the number of elements of $A$ that violate $A_{u v} \geq \underline{A}_{u v}^{\uparrow}$. As the number of elements of $A$ that violate $A_{i j} \leq \bar{A}_{i j}^{\downarrow}$ and $A_{u v} \geq \underline{A}_{u v}^{\uparrow}$ increases, the set of feasible solutions is reduced, thereby potentially increasing the attainable minimum cost.

\section{A. Robust Formulation of the Interval Observer Problem}

In the proposed robust formulation of Problem III.1, we define the inner bounds $\left(\bar{A}^{\downarrow}, \underline{A}^{\uparrow}\right)$ to be the nominal bounds on $A$. Thus, in the robust framework of Problem III.3, the inner bounds $\left(\bar{A}^{\downarrow}, \underline{A}^{\uparrow}\right)$ define the nominal constraint coefficients. Define $\Delta \bar{A}:=\bar{A}-\bar{A}^{\downarrow}, \Delta \underline{A}:=\underline{A}^{\uparrow}-\underline{A}$; these matrices define the perturbations to the coefficients in the robust LP. Coefficients corresponding to $\bar{A}_{i j}^{\downarrow}\left[\underline{A}_{i j}^{\uparrow}\right]$ are perturbed to $\bar{A}_{i j}^{\downarrow}+\Delta \bar{A}_{i j}=\bar{A}_{i j}\left[\underline{A}_{i j}^{\uparrow}-\Delta \underline{A}_{i j}=\underline{A}_{i j}\right]$.

We allow perturbations to be applied independently to each of the $2 n^{2}$ elements of $\left(\bar{A}^{\downarrow}, \underline{A}^{\uparrow}\right)$. This requires that the robust formulation of Problem III.1 have exactly one decision variable per element of $\bar{A}^{\downarrow}$ and $\underline{A}^{\uparrow}$. We introduce the variables $\bar{\lambda}, \underline{\lambda} \in \mathbb{R}^{n \times n}$, where $\bar{\lambda}_{i j}\left[\underline{\lambda}_{i j}\right]$ corresponds to $\bar{A}_{i j}^{\downarrow}$ $\left[\underline{A}_{i j}^{\uparrow}\right]$; perturbing the coefficient of $\bar{\lambda}_{i j}\left[\underline{\lambda}_{i j}\right]$ is interpreted as perturbing $\bar{A}^{\downarrow}$ upward $\left[\underline{A}^{\uparrow}\right.$ downward]. Define $\Lambda:=$ $\operatorname{col}(\bar{\lambda}, \underline{\lambda}) \in \mathbb{R}^{2 n^{2}}$. Since $\Lambda$ is $2 n^{2}$-dimensional, the sets of indices of uncertain coefficients is $J_{i} \subseteq \mathbf{2 n}^{2}, i \in\left(\mathbf{n}^{2}+\mathbf{n}\right)$, with cardinalities bounded by $\left|J_{i}\right| \leq n, i \in \mathbf{n}$, and $\left|J_{i}\right| \leq 1$, $i \in\left\{n+1, \ldots, n^{2}+n\right\}$.

Since the Hurwitz constraint of Problem III.1 is an equality, we characterize the effect of the protection process of [10] on equality constraints with uncertain coefficients.

Proposition IV.1. If constraint $i$ in Problem III.3 is an equality constraint with at least one uncertain coefficient $\left(J_{i} \neq \varnothing\right)$ and a nonzero protection level $\left(\Gamma_{i}>0\right)$, then Problem III.3 is infeasible.

Proof. An equality $\sum_{j} E_{i j} q_{j}=b_{i}$ can be formulated as two simultaneous inequalities: $\sum_{j} E_{i j} q_{j} \leq b_{i},-\sum_{j} E_{i j} q_{j} \leq$ $-b_{i}$. Let the index of the second constraint be $i^{\prime}$. The robust formulation is $\sum_{j} E_{i j} q_{j}+\Omega_{i}\left(q, \Gamma_{i}\right) \leq b_{i},-\sum_{j} E_{i j} q_{j}+$ $\Omega_{i^{\prime}}\left(q, \Gamma_{i^{\prime}}\right) \leq-b_{i}$. Adding the inequalities yields $\Omega_{i}\left(q, \Gamma_{i}\right)+$ $\Omega_{i^{\prime}}\left(q, \Gamma_{i^{\prime}}\right) \leq 0$, which is feasible only if $\Gamma_{i}=\Gamma_{i^{\prime}}=0$.

In light of Proposition III.3, in the robust version of Problem III.1 we must replace the equality Hurwitz constraint 
with an inequality. We propose the following cardinality constrained robust version of the IO design problem from [6].

\section{Problem IV.2.}

$$
\begin{aligned}
\text { minimize: } & {[2(\bar{A}-\underline{A}) \varkappa+\bar{\xi}-\underline{\xi}]^{\top} \lambda } \\
\text { subject to: } & \bar{A}_{i}^{\downarrow \top} \bar{\lambda}_{i}-C_{i}^{\top} Z \mathbf{1}_{n}+\Omega_{i}\left(\bar{\lambda}_{i}, \Gamma_{i}\right) \leq-1 \\
& -\left(\underline{A}_{j i}^{\uparrow} \underline{\lambda}_{j i}-C_{i}^{\top} Z_{j}+\delta_{i j} \beta\right)+\Omega_{r}\left(\underline{\lambda}_{i}, \Gamma_{r}\right) \leq 0 \\
& \lambda>\mathbf{0}_{n} \\
& \bar{\lambda}_{i}=\underline{\lambda}_{i}=\lambda \quad i, j \in \mathbf{n}, r=n i+j .
\end{aligned}
$$

Remark IV.3. If $p \leq n$, then the linear implementation of Problem IV. 2 has $\left(3 n^{2}+n(p+1)+1+\sum_{1}^{n^{2}+n}\left|J_{i}\right|\right) \in O\left(n^{2}\right)$ decision variables and constraints [10].

Let $J:=\left\{J_{1}, \ldots, J_{n^{2}+n}\right\}$ be the set of indices of uncertain coefficients, and $|J|:=\operatorname{col}\left(\left|J_{1}\right|, \ldots,\left|J_{n^{2}+n}\right|\right)$.

Theorem IV.4. If $\Gamma=|J|$, then Problems III.1 and IV.2 are equivalent.

To prove Theorem IV.4, we first prove that if $\Gamma=|J|$, then the coefficients in both problems are equal. We then prove that if Problem III.1 is feasible, then the Hurwitz constraint in Problem IV.2 is satisfied with equality at optimality.

Lemma IV.5. If $\Gamma=|J|$, then Problems III.1 and IV.2 have the same coefficients.

Proof. Lemma IV.5 follows from the definitions of $\bar{\lambda}, \underline{\lambda}, \bar{A}^{\downarrow}$, $\underline{A}^{\uparrow}$, and the perturbations (5) in Problem IV.2.

Proposition IV.6. Any feasible solution to Problem III.3 for given $\Gamma$, is also a feasible solution for any $\Gamma^{\prime} \preceq \Gamma$.

Proof. If $q$ is feasible in Problem III.3, then $\sum_{j=1}^{n} E_{i j} q_{j}+$ $\Omega_{i}\left(q, \Gamma_{i}\right) \leq b_{i}, i \in \mathbf{m}$. Since $\Gamma_{i}^{\prime} \leq \Gamma_{i}$ implies $\Omega\left(q, \Gamma_{i}^{\prime}\right) \leq$ $\Omega_{i}\left(q, \Gamma_{i}\right)$, we have $\sum_{j=1}^{n} E_{i j} q_{j}+\Omega_{i}\left(q, \Gamma_{i}^{\prime}\right) \leq \sum_{j=1}^{n} E_{i j} q_{j}+$ $\Omega_{i}\left(q, \Gamma_{i}\right) \leq b_{i}$.

By Lemma IV.5, if $\Gamma=|J|$, then Problems III.1 and IV.2 have equal coefficients, so any feasible solution to Problem III.1 is feasible in Problem IV.2 for $\Gamma \leq|J|$.

Lemma IV.7. If Problem III.1 is feasible and $\Gamma=|J|$, then the Hurwitz constraint of Problem IV.2 is satisfied with equality at optimality.

Proof. Let $(\lambda, Z, \beta)$ be optimal in Problem III.1. By Lemma IV.5, Problem IV.2 has the same coefficients and constraints as Problem III.1, except its Hurwitz constraint is an inequality instead of an equality. Therefore, the feasible region of Problem IV.2 is a superset of that of Problem III.1, thus $[2(\bar{A}-\underline{A}) \varkappa+\bar{\xi}-\underline{\xi}]^{\top} \lambda \geq[2(\bar{A}-\underline{A}) \varkappa+\bar{\xi}-\underline{\xi}]^{\top} \lambda^{\prime}$.

By Proposition IV.6, $(\bar{\lambda}, \operatorname{col}(\lambda, \ldots, \lambda), Z, \beta)$ is feasible in Problem IV.2, and satisfies its Hurwitz constraint with equality. For $\alpha>1$, the solution $(\alpha \lambda, \alpha \operatorname{col}(\lambda, \ldots, \lambda), \alpha Z, \alpha \beta)$ is feasible in Problem IV.2, and satisfies its Hurwitz constraint with inequality, and has cost $[2(\bar{A}-\underline{A}) \varkappa+\bar{\xi}-\xi]^{\top}(\alpha \lambda)>$ $[2(\bar{A}-\underline{A}) \varkappa+\bar{\xi}-\underline{\xi}]^{\top} \lambda$. Therefore, $(\lambda, \operatorname{col}(\lambda, \ldots, \lambda), Z, \beta)$ is optimal in Problem IV.2.

Proof of Theorem IV.4. By Lemma IV.5, if $\Gamma=|J|$, then Problems III.1 and IV.2 have the same coefficients, and by Lemma IV.7, the Hurwitz constraint of Problem IV.2 is satisfied with equality at optimality. Therefore, if $\Gamma=|J|$, then Problems III.1 and IV.2 are equivalent.

\section{B. Class of Systems Considered}

We now clarify the class of systems considered by Problem IV.2. Let the protection levels $\Gamma$ be fixed. Denote the set of subsets of $J_{i}$ with cardinality no greater than $\Gamma_{i}$ by $\mathcal{J}_{i}:=\left\{J_{i}^{\prime}\left|J_{i}^{\prime} \subseteq J_{i},\right| J_{i}^{\prime} \mid \leq \Gamma_{i}\right\}$, if $\Gamma_{i}=0$, then $\mathcal{J}_{i}:=\varnothing$; define the set of these sets $\mathcal{J}:=\left\{\mathcal{J}_{1}, \ldots, \mathcal{J}_{n^{2}+n}\right\}$. Let $S:=\left\{S_{1}, \ldots, S_{n^{2}+n}\right\}$ be an element of $\mathcal{J}$. Given pairs $\left(\bar{A}^{\downarrow}, \underline{A}^{\uparrow}\right),(\bar{A}, \underline{A}) \in \mathbb{R}^{n \times n} \times \mathbb{R}^{n \times n}$ satisfying Assumption 4 , define $\Xi: \mathcal{J} \rightarrow \mathbb{R}^{n \times n} \times \mathbb{R}^{n \times n}, S \mapsto\left(A^{u}, A^{l}\right)$, where $A_{j i}^{u}=\left\{\bar{A}_{j i}\right.$ if $i \leq n, j \in S_{i} ; \bar{A}_{j i}^{\downarrow}$ otherwise $\}, A_{j i}^{l}=$ $\left\{\underline{A}_{j i}\right.$ if $i, j \leq n, j \in S_{n i+j} ; \underline{A}_{j i}^{\uparrow}$ otherwise $\}$.

Using $\Xi$ we define the set-valued mapping $\mathcal{A}: \mathbb{Z}_{>0}^{n^{2}+n} \rightarrow$ $\mathbb{R}^{n \times n} \times \mathbb{R}^{n \times n}, \Gamma \mapsto\left\{\left(A^{u}, A^{l}\right):(\exists S \in \mathcal{J})(\Xi(S)=\right.$ $\left.\left.\left(A^{u}, A^{l}\right)\right)\right\} \cdot \mathcal{A}(\Gamma)$ is the set of perturbed $A$ matrix pairs $\left(A^{u}, A^{l}\right)$ for which the optimal solution to Problem IV.2 is deterministically feasible.

To solve Problem II.2 exactly, we must specify protection levels such that $\mathfrak{u}[\mathfrak{l}]$ coefficients defined by $\bar{A}^{\downarrow}\left[\underline{A}^{\uparrow}\right]$ are protected against perturbations. But this is not possible in the framework of Problem III.3, whose protection levels are specified per constraint.

\section{Robust Interval Observer Dynamics and Performance}

Assume Problem III. 1 is feasible. Let $\mathcal{L}(\Gamma)$ be the set of gain matrices $L$ constructed using optimal solutions to Problem IV.2 for a given $\Gamma$. By Theorem IV.4, the set of $L$ matrices generated by Problem III. 1 is $\mathcal{L}(|J|)$.

We propose the following robust interval observer (RIO), whose gain matrix (3) is constructed using an optimal solution to Problem IV.2.

$$
\begin{aligned}
& \dot{\hat{x}}^{u}=A^{u} \hat{x}^{u}+L\left(y-C \hat{x}^{u}\right)-\left(A^{u}-A^{l}\right) \phi\left(\hat{x}^{u}\right)+\bar{\xi} \\
& \dot{\hat{x}}^{l}=A^{u} \hat{x}^{l}+L\left(y-C \hat{x}^{l}\right)-\left(A^{u}-A^{l}\right) \psi\left(\hat{x}^{l}\right)+\underline{\xi} \\
& \left(\hat{x}^{u}(0), \hat{x}^{l}(0)\right)=\left(\bar{x}_{0}, \underline{x}_{0}\right), \quad\left(A^{u}, A^{l}\right) \in \mathcal{A}(\Gamma) .
\end{aligned}
$$

We now present our main result.

Theorem IV.8. Given a system of the form (1) satisfying Assumptions 1, 2, 3, and 4, and protection levels $\Gamma \preceq|J|$, if the proposed RIO (6) is constructed with $\left(A^{u}, A^{l}\right)=\Xi(S)$ such that $A^{l} \leq A \leq A^{u}$, then it results in a smaller upper bound on $\|\bar{e}\|_{1}$ than the IO (2).

Proof. By Lemma IV.5 and Proposition IV.6, the feasible region of Problem IV.2 is a superset of that of Problem III.1. Problems IV.2 and III. 1 have the same cost function, therefore the optimal cost of Problem IV.2 is less than or equal to that of Problem III.1. By (4), the cost upper bounds $\|\bar{e}\|_{1}$.

Remark IV.9. If only $x_{0}$ is uncertain, i.e., $\underline{A}=\bar{A}$ and $\xi(x, t) \equiv \xi(y, t)$, then an IO similar to (6) can be constructed [15], such that $\|\bar{e}\|_{1}=0$. An LP similar to Problem IV.2, is used to design the $L$ matrix. The observer's transient error is minimized, specifically $\int_{0}^{\infty}\|e(t)\|_{1} \mathrm{~d} t$. 


\section{Implementation}

We now delineate and illustrate the design process of the proposed RIO (6). 1) Identify $\bar{x}_{0}, \underline{x}_{0}, \bar{A}, \underline{A}, \bar{A}^{\downarrow}, \underline{A}^{\uparrow}, \varkappa$; 2) using the mapping $\Xi$ defined in Section IV-B, identify the elementwise smallest $\Gamma$ such that there exists a pair $\left(A^{u}, A^{l}\right) \in \mathcal{A}(\Gamma)$, such that $A^{l} \leq A \leq A^{u}$, for all possible system matrices $A$; 3 ) solve Problem IV.2 using the constraint $\lambda \geq \varepsilon \mathbf{1}_{n}$, where $\varepsilon>0$ is an arbitrarily small constant; 4) using the optimal $\lambda$ and $Z$, construct the observer gain matrix $\left.L:=\operatorname{diag}(\lambda)^{-1} Z^{\top} ; 5\right)$ construct the RIO (6) with $\left(A^{u}, A^{l}\right)$ from step 2 , and $L$ from step 4 .

Steps 2 and 5 minimize the attainable optimum cost while ensuring the interval property. The RIO is optimal in that the maximum cost, which upper bounds $\|\bar{e}\|_{1}$, over all system matrix pairs $\left(A^{u}, A^{l}\right) \in \mathcal{A}(\Gamma)$ is minimized.

For the class $\mathcal{A}(\Gamma)$, we solve Problem IV.2 once, i.e., design one $L$. The original IO (2) is guaranteed to be optimal for only $(\bar{A}, \underline{A})$, and would need to be optimized for each system matrix pair in $\mathcal{A}(\Gamma)$.

Example IV.10. To illustrate the proposed approach, we construct and implement a RIO for the system (1), with

$$
\begin{aligned}
A & =\left[\begin{array}{ccc}
-3.4 & 0.7 & 0.5 \\
0.7 & -3.4 & 1 \\
0.7 & 0.9 & -3.8
\end{array}\right] & C=\left[\begin{array}{lll}
0.9 & 0.4 & 0.6 \\
0.8 & 0.3 & 0.3
\end{array}\right] \\
\xi & =0.05 \sin \left(x^{\top} \mathbf{1}_{3}\right) \mathbf{1}_{3} & x_{0}=\left[\begin{array}{lll}
-2.5 & -1.1 & 1.3
\end{array}\right]^{\top} .
\end{aligned}
$$

We define the constants $\bar{x}_{0}=x_{0}+0.3\left|x_{0}\right|, \underline{x}_{0}=x_{0}-$ $0.3\left|x_{0}\right|, \bar{\xi}=-\underline{\xi}=(0.05) \mathbf{1}_{3}, \varkappa=(10.718) \mathbf{1}_{3}{ }^{2}$. Define $\bar{A}=A+0.4|A|, \underline{A}=A-0.4|A|, \bar{A}^{\downarrow}=A+0.1|A|$, $\underline{A}^{\uparrow}=A-0.1|A|$, thus yielding the cost vector $[2(\bar{A}-$ $\underline{A}) \varkappa+\bar{\xi}-\underline{\xi}]=\left[\begin{array}{lll}78.985 & 87.56 & 92.704\end{array}\right]^{\top}$. In Problem IV.2, only the coefficients of the variables $\bar{\lambda}$ and $\underline{\lambda}$, which are the elements of $\bar{A}^{\downarrow}$ and $\underline{A}^{\uparrow}$, respectively, are uncertain. The indices of uncertain coefficients are those corresponding to nonzero elements of $\Delta \bar{A}=\Delta \underline{A}=0.3|A|$. This yields $J_{1}=\{1,2,3\}, J_{2}=\{4,5,6\}, J_{3}=\{7,8,9\}, J_{i}=\{6+i\}$, $i \in\{4, \ldots, 12\}$. We set $\varepsilon=10^{-6}$.

Define $\kappa\left(L, A^{u}, A^{l}\right)$ to be the evaluation of (4), which upper bounds $\|\bar{e}\|_{1}$ of an IO with dynamics (6), if $A^{l} \leq$ $A \leq A^{u}$. We compare the objective values of the optimal solutions to Problems III.1 and IV.2, and the maximum bounds on $\|\bar{e}\|_{1}$ effected by the solutions to Problems III.1 and IV.2, as computed by (4), over the class $\mathcal{A}(\Gamma)$, i.e., $\left(1-\frac{\max _{\left(A^{u}, A^{l}\right) \in \mathcal{A}(\Gamma)} \kappa\left(L_{R I O}, A^{u}, A^{l}\right)}{\max _{\left(A^{u}, A^{l}\right) \in \mathcal{A}(\Gamma)} \kappa\left(L, A^{u}, A^{l}\right)}\right)$, where the fraction's numerator and denominator are the upper bound on $\|\bar{e}\|_{1}$ of (6) and (2), respectively, over $\mathcal{A}(\Gamma)$. The reductions in these values seen by using the proposed RIO (6), constructed with $\arg \max _{\left(A^{u}, A^{l}\right)} \kappa\left(L_{R I O}, A^{u}, A^{l}\right)$, over the IO (2), constructed with $\arg \max _{\left(A^{u}, A^{l}\right)} \kappa\left(L, A^{u}, A^{l}\right)$, i.e., each observer is constructed with the pair $\left(A^{u}, A^{l}\right)$ that maximizes its upper bound on $\|\bar{e}\|_{1}$ as computed by (4), are in Table I. Since $\Gamma$ is 12-dimensional, to facilitate presentation, we define the simulation parameters $\Gamma_{H}, \Gamma_{M} \in \mathbb{Z}_{\geq 0}$. The

\footnotetext{
${ }^{2}$ Derived using input-output stability theory [16, Corollary 5.2$]$
}

protection levels for the Hurwitz and Metzler constraints are set to $\Gamma_{H}$ and $\Gamma_{M}$, respectively, i.e., $\Gamma_{i}=\Gamma_{H}, i \in$ $\{1,2,3\}$, and $\Gamma_{i}=\Gamma_{M}, i \in\{4, \ldots, 12\}$. Plots of the trial $\Gamma_{H}=3, \Gamma_{M}=0$ are in Figure 1, where the proposed RIO exhibits improved steady-state and transient behaviour over the original IO on their respective worst-case $\left(A^{u}, A^{l}\right)$.

TABLE I

IMPROVEMENT IN WORST-CASE VALUES (\%) FOR EXAMPLE IV.10.

\begin{tabular}{|c|c|c|c|c|}
\hline$\Gamma_{H}$ & $\Gamma_{M}$ & Cost & Bound & $\|\bar{e}\|_{1}$ \\
\hline 0 & 1 & 43.8 & 43.9 & 0.00 \\
\hline \multirow{2}{*}{1} & 0 & 36.7 & 41.1 & 28.6 \\
\cline { 2 - 5 } & 1 & 14.8 & 18.0 & 3.73 \\
\hline \multirow{2}{*}{2} & 0 & 30.0 & 33.0 & 31.0 \\
\cline { 2 - 5 } & 1 & 2.34 & 4.69 & 3.23 \\
\hline 3 & 0 & 28.6 & 28.8 & 29.0 \\
\hline
\end{tabular}

In Table I, the reductions in optimal cost, bounds on $\|\bar{e}\|_{1}$ and $\|\bar{e}\|_{1}$ over the class $\mathcal{A}(\Gamma)$ are as high as $43.9 \%, 43.9 \%$ and $31.0 \%$, respectively.

\section{Monte CARlo Analysis}

We conduct a Monte Carlo analysis to characterize the performance improvement seen by using the proposed RIO (6) instead of the IO (2).

The elements of the matrices $\bar{A}, \underline{A}, \bar{A}^{\downarrow}, \underline{A}^{\uparrow} \in \mathbb{R}^{n \times n}$ are seeded by uniform random variables on the intervals $\widetilde{A}_{i j} \in$ $[0,1], i, j \in \mathbf{n}, i \neq j, \widetilde{A}_{i i} \in[-2 n,-n], i \in \mathbf{n}$, which ensures the Hurwitz property. The seed matrix $\widetilde{A} \in \mathbb{R}^{n \times n}$ is modified to generate the system matrices such that $\|\bar{A}-\underline{A}\|_{1}=1$, which provides consistency in the cost coefficients across trials, and $\bar{A}^{\downarrow}-\underline{A}^{\uparrow} \geq \frac{2}{n^{3}} \mathbf{1}_{n \times n}$, which precludes constraint coefficients being arbitrarily close to 0 . The matrix $C \in$ $\mathbb{R}^{p \times n}$ is generated as a uniform random variable on the interval $C \in[0,1]^{p \times n}$. The initial conditions $\bar{x}_{0}, \underline{x}_{0} \in \mathbb{R}^{n}$ are seeded by the uniform random variable $x_{0} \in[-n, n]^{n}$, and are set to $\bar{x}_{0}=x_{0}+\frac{1}{2 n} \mathbf{1}_{n}, \underline{x}_{0}=x_{0}-\frac{1}{2 n} \mathbf{1}_{n}$, which guarantees $\left\|\bar{x}_{0}-\underline{x}_{0}\right\|_{1}=1$. We define the constants $\bar{\xi}=-\underline{\xi}=(0.1) \mathbf{1}_{n}$, $\varkappa=2 \max \left(\left|\bar{x}_{0}\right|,\left|\underline{x}_{0}\right|\right)$, where max is taken elementwise. We simulate the dynamical system $\dot{x}=A x+\delta \sin \left(x^{\top} \mathbf{1}_{n}\right) \mathbf{1}_{n}$, $y=C x$, where $\delta$ is generated as a uniform random variable on the interval $\delta \in[-0.1,0.1]$. This system can be viewed as a stable LTI system $\dot{x}=A x+B u$, where $B:=I$ and $|u(t)| \leq|\delta|$, which satisfies Assumption 1. The system is simulated for $t \in[0,100]$ using MATLAB's ode $15 \mathrm{~s}$. The plant is assumed to be at steady-state at $t=100$. If $\varkappa \preceq \max _{t \in[0,100]}|x(t)|$, or if Problem III.1 is infeasible, then the trial is discarded.

Due to the potentially large dimension of $\Gamma$, we define the simulation parameter $\Gamma^{\star} \in \mathbb{Z}_{>0}$. The protection levels for the Hurwitz constraints are set to $\Gamma_{i}=\min \left(\Gamma^{\star},\left|J_{i}\right|\right)$, $i \in \mathbf{n}$, and a randomly populated set $\mathcal{I} \subseteq\left\{n+1, \ldots, n^{2}+\right.$ $n\}$ of cardinality $n \Gamma^{\star}$, contains the indices of the Metzler constraints whose protection levels are set to 1 . Thus $n \Gamma^{\star}$ elements of $\bar{A}^{\downarrow}$, and $n \Gamma^{\star}$ elements of $\underline{A}^{\uparrow}$, are perturbed in each trial.

Ten thousand trials are conducted for representative combinations of $n, p$, and $\Gamma^{\star}$. In each trial, Problems III.1 

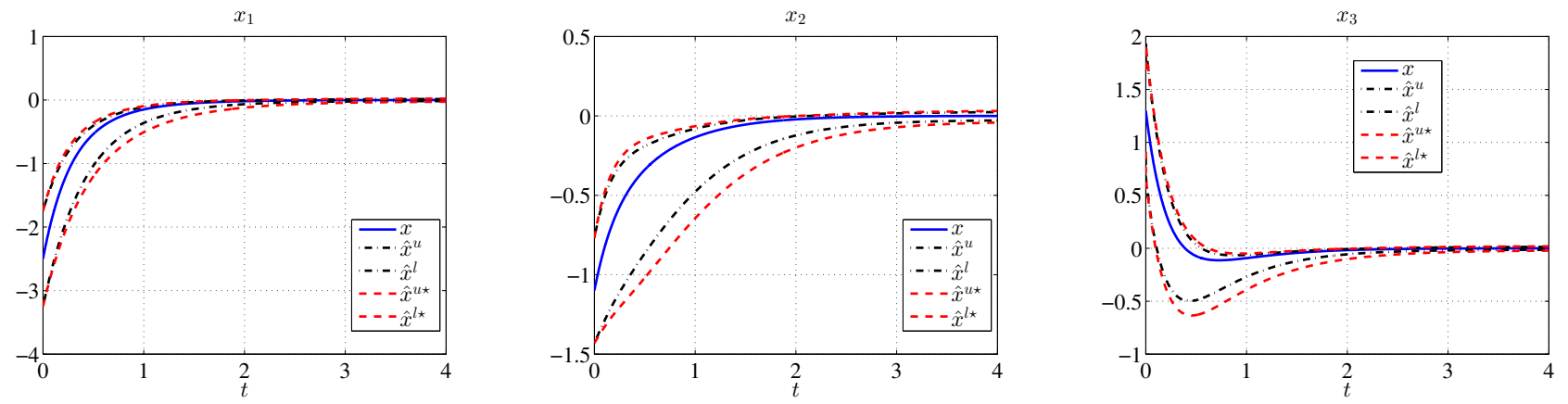

Fig. 1. RIO $\hat{x}$ and original IO $\hat{x}^{\star}$ constructed with their respective worst-case $\left(A^{u}, A^{l}\right)$ over $\mathcal{A}\left(\operatorname{col}\left(3,3,3, \mathbf{0}_{9}\right)\right)$.

and IV.2 are solved for the same parameters, and the percent differences between their costs, worst-case costs over the class $\mathcal{A}(\Gamma)$, i.e., $100\left(1-\frac{\max _{\left(A^{u}, A^{l}\right) \in \mathcal{A}(\Gamma)} \kappa\left(L_{R I O}, A^{u}, A^{l}\right)}{\max _{\left(A^{u}, A^{l}\right) \in \mathcal{A}(\Gamma)} \kappa\left(L, A^{u}, A^{l}\right)}\right)$, and true $\|\bar{e}\|_{1}$ values on the worst-case $\left(A^{u}, A^{l}\right)$ are recorded. These metrics' means $\mu$ and standard deviations $\sigma$ are in Table II.

TABLE II

IMPROVEMENT IN COST, WORST-CASE BOUND, AND $\|\bar{e}\|_{1}(\%)$.

\begin{tabular}{|c|c|c|c|c|c|c|c|c|}
\hline \multirow[b]{2}{*}{$n$} & \multirow[b]{2}{*}{$p$} & \multirow{2}{*}{$\Gamma^{\star}$} & \multicolumn{2}{|c|}{ Cost } & \multicolumn{2}{|c|}{ Bound } & $\bar{e}$ & 1 \\
\hline & & & $\mu$ & $\sigma$ & $\mu$ & $\sigma$ & $\mu$ & $\sigma$ \\
\hline 2 & \multirow{3}{*}{1} & \multirow{3}{*}{1} & 4.20 & 6.06 & 4.18 & 6.00 & 3.17 & 5.66 \\
\hline 3 & & & 6.30 & 7.09 & 6.29 & 7.09 & 4.42 & 6.74 \\
\hline 4 & & & 6.81 & 7.63 & 6.81 & 7.64 & 4.74 & 7.37 \\
\hline \multirow{4}{*}{5} & 1 & \multirow{4}{*}{1} & 6.82 & 7.56 & 6.83 & 7.58 & 4.77 & 7.28 \\
\hline & 2 & & 4.56 & 1.90 & 4.59 & 1.96 & 2.77 & 2.58 \\
\hline & 3 & & 3.24 & 1.43 & 3.33 & 1.69 & 2.18 & 2.72 \\
\hline & 4 & & 1.27 & 0.944 & 1.37 & 1.48 & 1.02 & 3.57 \\
\hline \multirow{3}{*}{5} & \multirow{3}{*}{4} & 2 & 0.776 & 0.811 & 0.835 & 1.12 & 0.784 & 3.85 \\
\hline & & 3 & 0.511 & 0.626 & 0.520 & 0.753 & 0.430 & 6.85 \\
\hline & & 4 & 0.267 & 0.480 & 0.280 & 0.624 & 0.257 & 3.23 \\
\hline
\end{tabular}

The improvements in all three metrics correlate negatively with the protection levels $\Gamma^{\star}$ and number of outputs $p$, and positively with the number of states $n$. The proposed RIO (6) is most effective when $p$ is much smaller than $n$. Thus, the proposed RIO is a more attractive solution for more complex plants, i.e., large $n$, and when few measurements are available, i.e., $p$ is low. Lastly, the standard deviation exceeds the mean for most parameter combinations, suggesting the performance of the RIO is highly plant-dependent.

\section{CONCLUSIONS}

We applied the robust optimization method of [10] to the linear programming-based IO design procedure of [6] to solve an approximation of Problem II.2. We proved that the cost of the proposed RIO design LP is strictly less than that of the original IO. The Monte Carlo analysis suggests that the cost reduction effected by the proposed RIO correlates positively with the number of states, and negatively with the number of outputs. However, the standard deviations of the cost reduction were high, indicating that the reduction is highly dependent upon the specific system being observed.

Future work should identify a method of coupling specific cost and constraint perturbations, as this would allow for optimality and feasibility to be guaranteed over the same class of systems. Analytic bounds on the cost reduction effected by using the proposed RIO over the IO of [6] should be identified. Also, an objective function should be identified for simultaneous optimization of transient and steady-state performance.

\section{REFERENCES}

[1] J.-L. Gouzé, A. Rapaport, and M. Z. Hadj-Sadok, "Interval observers for uncertain biological systems," Ecological Modelling, vol. 133, no. $1-2,2000$.

[2] G. Goffaux, A. Vande Wouwer, and O. Bernard, "Improving continuous-discrete interval observers with application to microalgaebased bioprocesses," Journal of Process Control, vol. 19, no. 7, pp. 1182-1190, 2009.

[3] M. A. Rami, F. Tadeo, and U. Helmke, "Positive observers for linear positive systems, and their implications," International Journal of Control, vol. 84, no. 4, 2011.

[4] F. Mazenc and O. Bernard, "Interval observers for linear time-invariant systems with disturbances," Automatica, vol. 47, no. 1, 2011.

[5] Z. Shu, J. Lam, H. Gao, B. Du, and L. Wu, "Positive Observers and Dynamic Output-Feedback Controllers for Interval Positive Linear Systems," IEEE Trans. Circuits Syst. Regul. Pap., vol. 55, no. 10, 2008.

[6] M. A. Rami, C. Cheng, and C. de Prada, "Tight robust interval observers: An LP approach," in IEEE Conference on Decision and Control, Cancun, Mexico, 2008, pp. 2967-2972.

[7] D. Bertsimas, D. B. Brown, and C. Caramanis, "Theory and Applications of Robust Optimization," SIAM Review, vol. 53, no. 3, pp. 464-501, 2011.

[8] A. Ben-Tal and A. Nemirovski, "Robust solutions of uncertain linear programs," Operations Research Letters, vol. 25, no. 1, pp. 1-13, 1999.

[9] D. Bertsimas, D. Pachamanova, and M. Sim, "Robust linear optimization under general norms," Oper. Res. Letters, vol. 32, no. 6, 2004.

[10] D. Bertsimas and M. Sim, "Robust discrete optimization and network flows," Mathematical Programming, vol. 98, no. 1-3, pp. 49-71, 2003.

[11] A. Ben-Tal and A. Nemirovski, "Robust optimization - methodology and applications," Math. Program., vol. 92, no. 3, pp. 453-480, 2002.

[12] S. P. Boyd, S.-J. Kim, D. D. Patil, and M. A. Horowitz, "Digital Circuit Optimization via Geometric Programming," Operations Research, vol. 53, no. 6, pp. 899-932, 2005.

[13] D. Bertsimas and D. B. Brown, "Constrained Stochastic LQC: A Tractable Approach," IEEE Transactions on Automatic Control, vol. 52, no. 10, pp. 1826-1841, 2007.

[14] K.-L. Hsiung, S.-J. Kim, and S. P. Boyd, "Power control in lognormal fading wireless channels with uptime probability specifications via robust geometric programming," in Proceedings of the American Control Conference, Portland, OR, 2005, pp. 3955-3959.

[15] P. J. McCarthy, "Cardinality Constrained Robust Optimization Applied to a Class of Interval Observers," M.A.Sc. thesis, University of Waterloo, 2013. [Online]. Available: http://hdl.handle.net/10012/7907

[16] H. K. Khalil, Nonlinear Systems, 3rd ed. Upper Saddle River, NJ: Prentice Hall, 2002. 